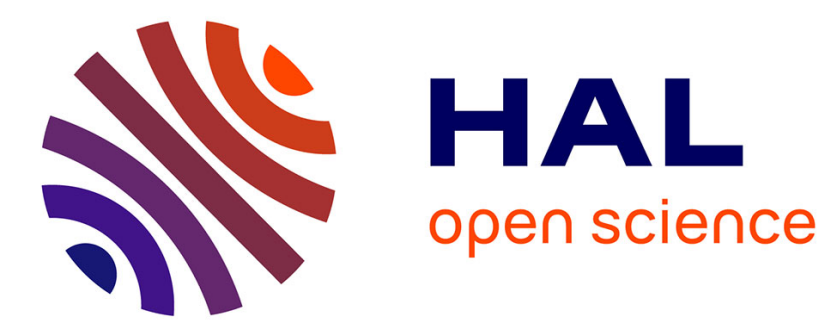

\title{
The Magnetic Aspects of the $\gamma-\alpha$ and $\gamma-\epsilon$ Martensitic Transformations in Fe-Mn Alloys
}

\author{
M. Acet, T. Schneider, B. Gehrmann, E. Wassermann
}

\section{To cite this version:}

M. Acet, T. Schneider, B. Gehrmann, E. Wassermann. The Magnetic Aspects of the $\gamma-\alpha$ and $\gamma-\epsilon$ Martensitic Transformations in Fe-Mn Alloys. Journal de Physique IV Proceedings, 1995, 05 (C8), pp.C8-379-C8-384. 10.1051/jp4:1995856 . jpa-00254105

\section{HAL Id: jpa-00254105 https://hal.science/jpa-00254105}

Submitted on 1 Jan 1995

HAL is a multi-disciplinary open access archive for the deposit and dissemination of scientific research documents, whether they are published or not. The documents may come from teaching and research institutions in France or abroad, or from public or private research centers.
L'archive ouverte pluridisciplinaire HAL, est destinée au dépôt et à la diffusion de documents scientifiques de niveau recherche, publiés ou non, émanant des établissements d'enseignement et de recherche français ou étrangers, des laboratoires publics ou privés. 


\title{
The Magnetic Aspects of the $\gamma-\alpha$ and $\gamma-\in$ Martensitic Transformations in Fe-Mn Alloys
}

\author{
M. Acet, T. Schneider, B. Gehrmann* and E.F. Wassermann \\ Tieftemperaturphysik, Gerhard-Mercator Universität Duisburg, 47048 Duisburg, Germany \\ * Krupp VDM GmbH, 58778 Werdohl, Germany
}

\begin{abstract}
Fe}_{100-x} \mathrm{Mn}_{\mathrm{x}}$ alloys undergo fcc-bcc $(\gamma-\alpha)$ and fcc-hcp $(\gamma-\epsilon)$ transformations for about $0<\mathrm{x}<10$ at $\%$ and $15<\mathrm{x}<30$ at $\%$ respectively. The product phase in the region $10<\mathrm{x}<15$ at. $\%$ is mixed $\alpha+\epsilon$ and $\epsilon+\gamma$. The $\gamma-\alpha$ transformation in $\mathrm{Fe}-\mathrm{Mn}$ is similar to that in Fe-rich $\mathrm{Fe}-\mathrm{Ni}$ alloys where the transformation takes place between a high-temperature dense fcc structure and a lowtemperature more open bcc structure. This anomalous volume increase at the martensite transformation temperature is closely related to short-range ferromagnetic correlations in the fcc and bcc states. The $\gamma-\epsilon$ transformation in Fe-Mn takes place between a high-temperature fcc structure and a relatively denser packed low-temperature hcp structure. In this case there is no anomalous volume increase.In the present work we investigate the volumetric properties of $\mathrm{Fe}_{100-\mathrm{x}} \mathrm{Mn}_{\mathrm{x}}$ with $\mathrm{x}=8$ 30 at $\%$ and examine the influence of the magnetic properties on the $\gamma-\alpha$ and $\gamma-\epsilon$ martensitic transformations. Results show that the $\gamma-\epsilon$ transformation occurs with the simultaneous loss of ferromagnetic correlations in the $\gamma$-phase
\end{abstract}

\section{INTRODUCTION}

The equilibrium ground-state of fcc $\mathrm{Fe}(\gamma-\mathrm{Fe})$ and fcc $\mathrm{Mn}(\gamma-\mathrm{Mn})$ are predicted to be antiferromagnetic (AF). If the volume is expanded beyond a critical value the coupling becomes ferromagnetic (FM) [1-3]. Such a property is an example of a moment-volume-instability which is the cause of modified anharmonicity leading to the Invar and anti-Invar effects observed in many fcc $3 \mathrm{~d}$-alloys incorporating Fe and $\mathrm{Mn}[4,5]$. Both $\gamma-\mathrm{Fe}$ and $\gamma-\mathrm{Mn}$ have limited stability ranges of $1184-1665 \mathrm{~K}$ and $1373-1411 \mathrm{~K}$ respectively. However, when alloyed together to form $\mathrm{Fe}_{100-\gamma} \mathrm{Mn}_{v}$, they open up eachother's $\gamma$-phase and stablize it in the complete temperature range in the concentration interval $30<x<60$ at. $\%[6]$. These alloys have been extensively studied because of their antiferromagnetic (AF) Invar and anti-Invar properties $[7,8]$. For concentrations of roughly $\mathrm{x}<10$ at. \% Fe-Mn alloys undergo an fcc-bcc $(\gamma-\alpha)$ martensitic transformation. For about $10<\mathrm{x}<30$ at. $\%$ the martensitic transformation takes the form of fcc-hcp $(\gamma-\epsilon)$ [9]. These alloys are of particular technical importance because, by the addition of a third element such as Si the $\gamma-\epsilon$ transformation can be exploited for the shape memory effect [10-12].

When it comes to dealing with martensitic transformations in $\mathrm{Fe}$ alloys, Fe becomes the central issue with the crucial question "why is the high temperature $\gamma$-phase denser packed than the low temperature $\alpha$ phase?" In a recent work we argued that the anomalous volume increase at the $A_{3}$ point $(1184 \mathrm{~K})$ must be related to an interplay between magnetic and lattice vibrational properties in the $\gamma$-phase [13]. $\gamma$-Fe is an antiInvar, i.e., the ground state is AF but the magnetic correlations in the stability range are FM [4]. The argument suggests that with decreasing temperature the strength of FM correlations in the $\gamma$-phase increase due to decrease of lattice vibrational amplitude, while at the same time the volume decreases. This leads to an increase of internal pressuı until the volume can no longer support the fcc structure, and a transformation to the more open bcc structure is triggered. Energy analysis shows that the stabilization of the $\alpha$-phase of Fe is 
established by the presence of ferromagnetism. This does not necessarily have to be in the form of long range FM order since the $A_{3}$ point lies above $T_{C}=1041 \mathrm{~K}$ in the paramagnetic (PM) range. Therefore, the existence of FM correlations are sufficient for the stabilization. Without ferromagnetism in the $\alpha$-phase $\gamma$-Fe would have been stable down to about $500 \mathrm{~K}$ below which hcp Fe $(\epsilon-\mathrm{Fe})$ would have been stable [14]. Pure $\epsilon$-Fe can only be stabilized under a pressure of about $13 \mathrm{GPa}$ at room temperature.

The stabilization of the $\alpha$-phase and therefore the cause of the $\gamma$ - $\alpha$ martensitic transformation in Fe alloys such as Fe-Ni, Fe-Pt and Fe-Pd is expected to be of similar origin. Another class of Fe alloys such as Fe-Mn, $\mathrm{Fe}-\mathrm{Ir}$ and $\mathrm{Fe}-\mathrm{Ru}$, which in addition to the $\gamma-\alpha$ transformation on the Fe-rich side, also undergo a $\gamma-\epsilon$ transformation in an $\mathrm{Fe}$ concentration region of $\sim 75-90$ at. \% [9]. In these alloys $\mathrm{Fe}$ can be stabilized in an hcp environment without the necessity of external pressure. Just exactly what causes this transformation in a certain class of $\mathrm{Fe}$ alloys and in others not is a subject of current research [15]. In the present paper we examine the $\gamma-\alpha$ and $\gamma-\epsilon$ martensitic transformations in Fe-Mn alloys by thermal expansion measurements and construct the phase diagram. We examine the features of this phase diagram together with the magnetic properties and discuss the mechanisms that are responsible for the $\gamma-\alpha$ and $\gamma-\epsilon$ martensitic transformations.

\section{EXPERIMENT}

$\mathrm{Fe}_{100-x} \mathrm{Mn}_{\mathrm{x}}$ alloys with $\mathrm{x}=8,10,12,15$ and 25 at. \% were prepared by arc melting under $\mathrm{Ar}$ atmosphere. Prior to measurements the samples were maschined to $7 \mathrm{~mm}$ long and $6 \mathrm{~mm}$ diameter cylinders. They were then annealed in their austenite phase for two hours and furnace cooled to room temperature. The samples were checked at room temperature by X-ray measurements for the $\alpha, \gamma$ and $\epsilon$ phases. The results are summarized in Tab. 1. The thermal expansion $\Delta 1 / 1$ was measured in two separate dilatometers covering the temperature ranges $4<T<300 \mathrm{~K}$ and $300<\mathrm{T}<1200 \mathrm{~K}$ respectively. The samples were first measured from room temperature to $4 \mathrm{~K}$ and back, and then from room temperature up to about $1200 \mathrm{~K}$ and down. Data were taken at equilibrium in $3 \mathrm{~K}$ and $5 \mathrm{~K}$ intervals in the low temperature and high temperature apparatus respectively.

\section{RESULTS}

\subsection{Thermal expansion}

Fig. 1 shows $\Delta \mathrm{l} / \mathrm{l}$ vs $\mathrm{T}$ of all five alloys. The alloys with $x=8,10$ and 12 at. \% show a broad hysteresis with the cooling curve lying lower than the heating curve. The increase of volume at the martensite start temperature $M_{s}$ with decreasing temperature shows that the product phase of these alloys is $\alpha$-dominated (subscripts "s" and " $\mathrm{f}$ " refer to start and finish). This is also confirmed by $X$-ray measurements which show only $\alpha$-phase for $x=8$ and 10 at $\%$ and traces of $\epsilon$ for $x=12$ at $\%$. There are also some fine structures observable just below the austenite finish ( $\left.A_{f}\right)$ temperatures. As $A_{f}$ is approached with increasing temperature $\Delta V / 1$ first tends to level off showing a slowing down of the formation of austenite. It then decreases again abruptly as the formation is reaccellerated, and eventually merges with the cooling curve. The hysteresis in the curves for $x=15$ and 25 at. $\%$ is reversed, i. e., the cooling curve lies above the

Tab. 1. The phases of Fe 100 . Mn observed at room temperature by X-ray analysis. Inderlining denotes dominating phase.

\begin{tabular}{|l|c|c|c|c|c|c|}
\hline$x($ at. \%) & 8 & 10 & 12 & 15 & 25 & 30 \\
\hline phase & $\alpha$ & $\alpha$ & $\underline{\alpha}+\epsilon$ & $\alpha+\epsilon$ & $\gamma+\epsilon$ & $\gamma$ \\
\hline
\end{tabular}

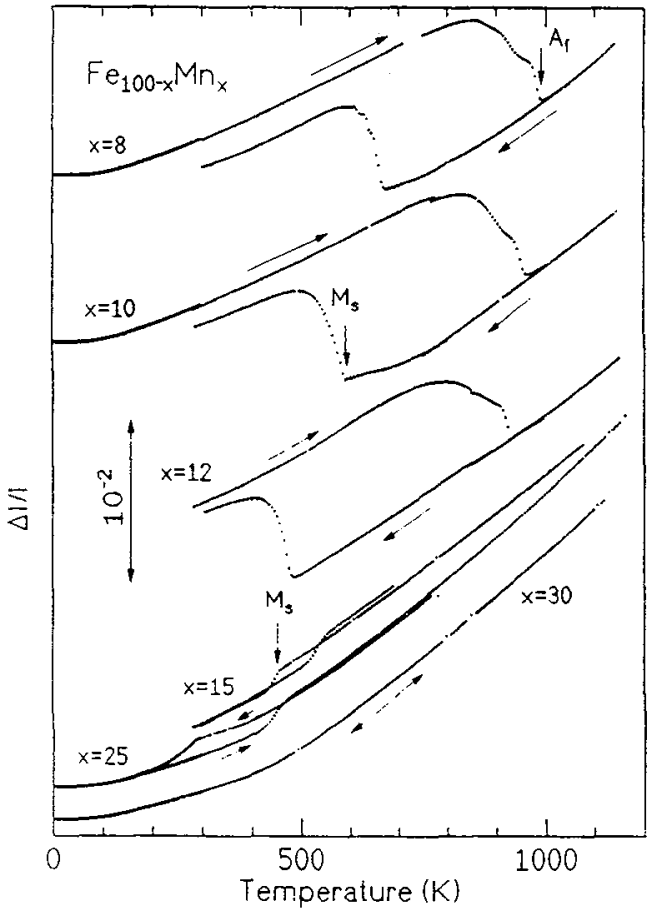

1.ig. 1. The temperature dependence of the thermal expansion of $\mathrm{Fe}_{10-x} \mathrm{Mn}$. for $8<\mathrm{x}<30$ at. \%. Alloys with $\mathrm{s}=8-12$ at. \% show a volume increase at $M$ Thosc with $\mathrm{x}=15$ and 25 at. \% show a rolume decrease. The arrows indicate the measurement dircetion. 

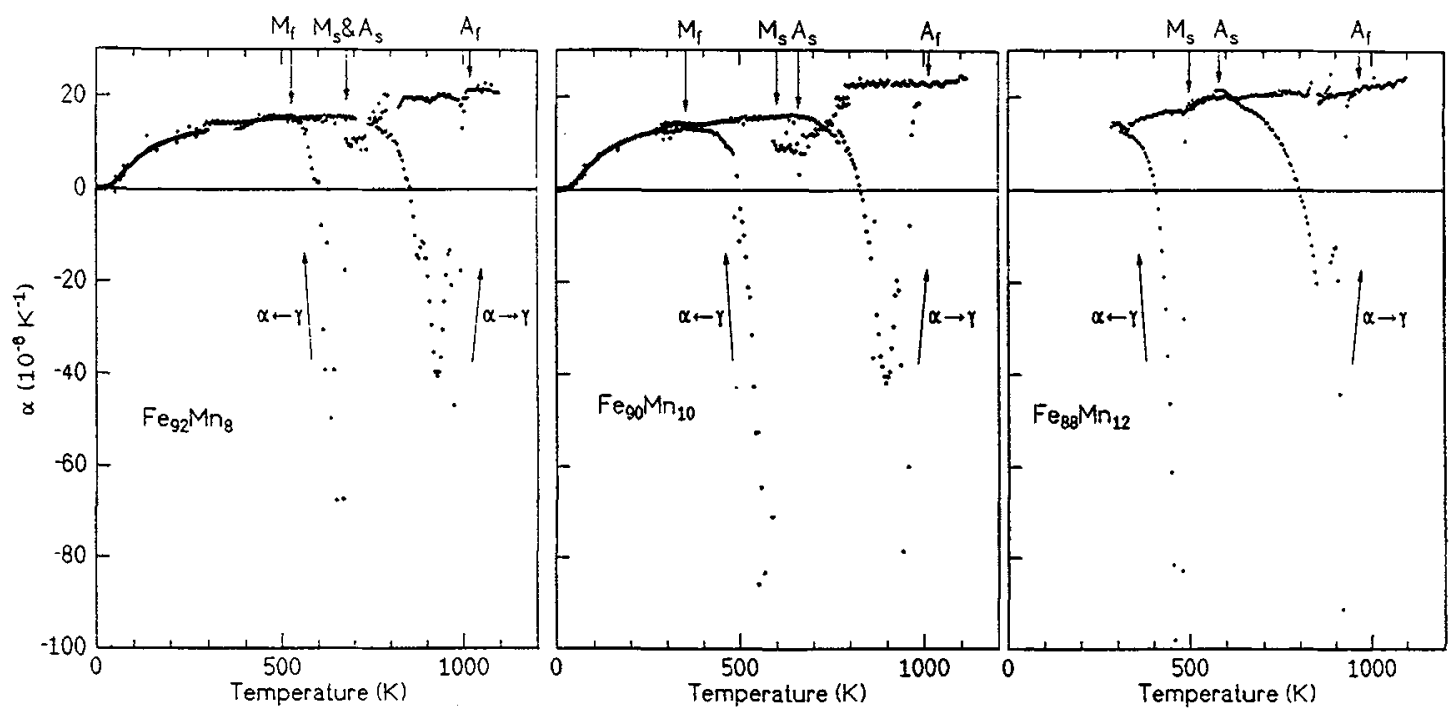

Fig. 2. $\alpha$ \&s $\mathrm{T}$ for $\mathrm{x}=8,10$ and 12 at. $\%, \mathrm{M}_{s}, \mathrm{M}_{\mathrm{f}}, \mathrm{A}_{\mathrm{s}}$ and $\mathrm{A}_{\mathrm{f}}$ are indicated by arrows on the upper scale.

heating curve. There is a volume decrease at $M_{s}$ showing that the product phase is $\epsilon$-dominated. $X$-ray measurements confirm that this phase is composed of mainly $\epsilon$ with only traces of $\alpha$. The $x=30$ at. \% sample shows no transformation down to the lowest temperatures. X-ray measurements for this sample also show only a $\gamma$-phase.

Another feature seen in Fig. 1 is that the hysteresis of the curves do not close. This is due to the different amount of martensite at the begining of the experiment, where the samples during preparation are furnacecooled at a rate of $\sim 5 \mathrm{~K} / \mathrm{min}$, and at the end, where the sample is measured on cooling from $1200 \mathrm{~K}$ downwards at a relatively slower effective rate of $\sim 0.4 \mathrm{~K} / \mathrm{min}$.

Fig 2 shows the temperature dependence of the thermal expansion coefficient $\alpha(T)$ for $x=8,10$ and 12 at. $\%$. The curves show typical features of an $\gamma-\alpha$ transformation and are similar to those reported earlier for FeNi alloys [9]. $M_{s}, M_{f}, A_{s}$, and $A_{f}$ are shown by arrows. The positions of these temperatures are chosen as the point where the curves deviate from regular behavior near the spikes.
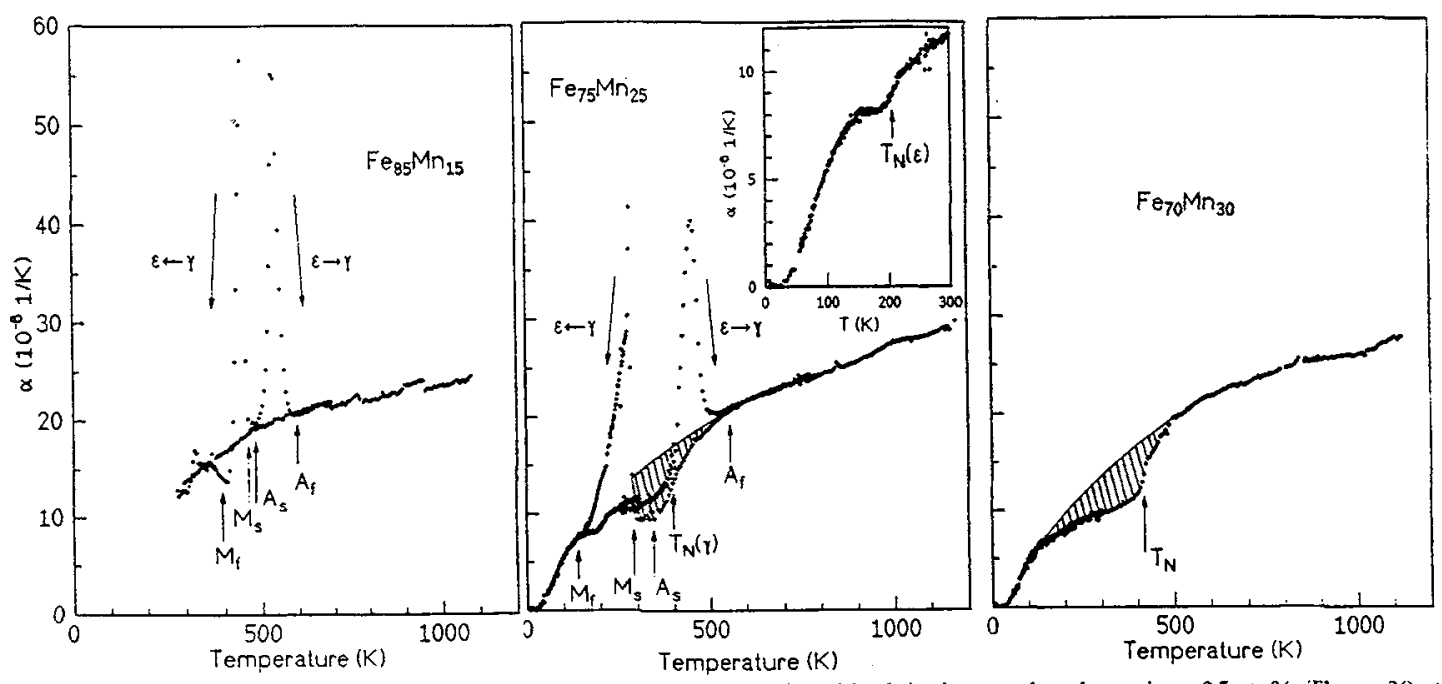

Fig. 3. \& $\& \mathrm{~s} \mathrm{~T}$ of $\mathrm{x}=15.25$ and 30 at $\%$ Magnetovolume effects are found both in the $\gamma$ and $\epsilon$-phases in $\mathrm{x}=25$ at. $\%$. The $\mathrm{x}=30$ at. "osample is only $\gamma$-phase and shows a magnitowinme effect. $T_{V}(\gamma)$ and $T_{\Upsilon}(\epsilon)$ are the Néel temperatures of the $\gamma$ and $\epsilon-$ phases respectively. Inset in the middle figure shows magnetovolume effect of the $\epsilon$-phase of $x=25$ at. $\%$. 
Fig. 3 shows $\alpha$ vs. $T$ for $x=15,25$ and 30 at. $\%$. The structural transformation temperatures are likewise shown in the figures. The $\mathrm{x}=15$ at. \% sample shows regular behavior other than a slight dip near $\mathrm{M}_{\mathrm{f}}$. The exact nature of this feature is not known, however it can be due to either a continuation of the transition or a small magnetovolume effect associated with AF-ordering of the coexisting $\gamma$-phase. Both the pure $\gamma$-phase sample, $\mathrm{x}=30$ at. $\%$, and the $\gamma$-phase of $\mathrm{x}=25$ at. $\%$ order antiferromagneticaly around $\mathrm{T}_{\mathrm{N}}(\gamma)$ and exhibit magnetovolume effects, shown by the hatched areas, typical of fcc Fe-Mn alloys [8]. However, for $\mathrm{x}=25$ at. $\%$ we observe an additional magnetovolume effect around a temperature which almost coincides with the Néel temperature, $T_{N}(\epsilon) \simeq 230 \mathrm{~K}$, of the $\epsilon$-phase [16]. This feature which is shown in the inset is expected to be due to magnetic ordering in the $\epsilon$ phase.

\subsection{The phase diagram}

The transformation temperatures obtained from the present $\alpha(\mathrm{T})$ data are shown in Fig. 4. The $\alpha-\gamma$ transformation curves merge at the $\mathrm{A}_{3}$ point of $\mathrm{Fe}$. The structures observed by $\mathrm{X}$-rays in the product phases are also shown. Between $\mathrm{x}=10$ and 15 at $\% \mathrm{Mn}$ the amount of $\epsilon$-phase formed depends strongly on concentration. Although a pure $\epsilon-$ phase is expected to be stable above a concentration where $\alpha$ totally disappears, according to [16], between 17.8 and 28.6 at. $\% \mathrm{Mn}$ traces of the $\gamma$-phase seem to be inevitable even under the most stringent sample preparation conditions. It is therefore difficult to establish experimentally the exact boundaries of the phases. The boundaries are shown instead with some uncertainty.

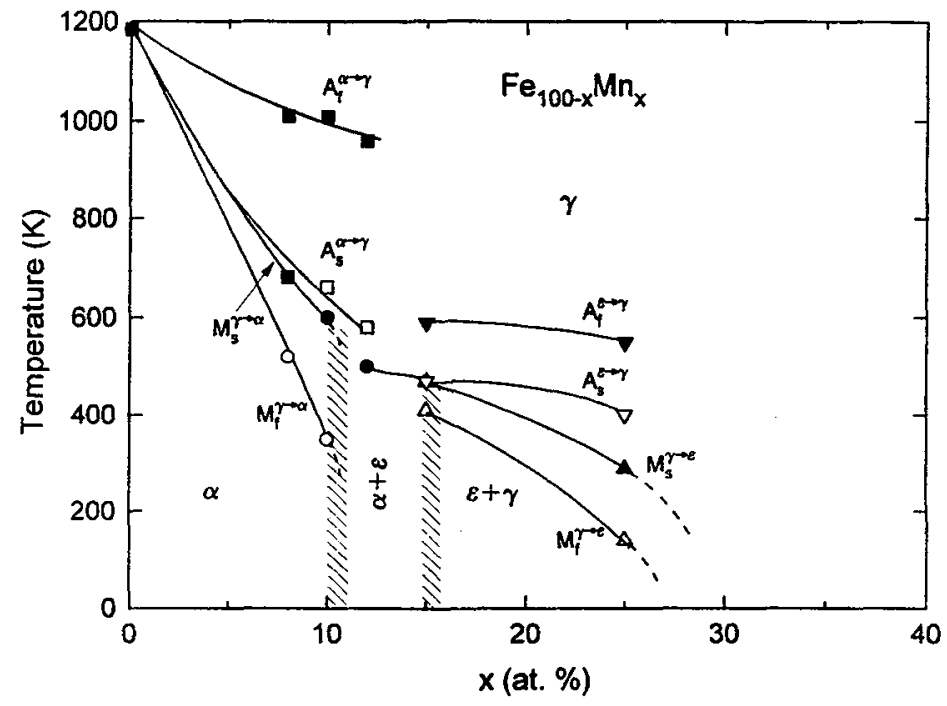

Fig. 4. The martensite and austenite transformation temperatures of Fe-Mn obtained from the present data. The $\alpha+\epsilon$ phase is such that near $x=10$ at. $\%$ it is $\alpha$ dominant, and near $x=15$ at. $\%$ it is $\epsilon$-dominant.

\section{DISCUSSION}

The properties of martensitic transformations of Fe alloys are closely related to their magnetic properties. In order to understand this relationship in Fe-Mn we plot in Fig. 5 the $\mathrm{M}_{s}$ temperatures together with the magnetic transformation temperatures. The $\alpha$-phase is FM with the Curie temperatures denoted by $\mathrm{T}_{C}(\alpha)$, whereas the magnetic coupling in both the $\epsilon$ and $\gamma$-phases is AF with Néel temperatures denoted by $T_{\vee}(\epsilon)$ and $T_{\aleph}(\gamma)$ respectively [16-19]. $T_{f}(\gamma)$ denotes the freezing temperatures below which the system enters into a frustrated spin-glass-like state [18]. In the $\epsilon$-phase of pure Fe there is no long range magnetic order, i.e., $T_{\vee}(\epsilon-F e)=0[20]$. Total energy calculations for $\epsilon-\mathrm{Fe}$ have shown that at slightly expanded volumes $\mathrm{AF}$ ordering sets in [21]. Therefore, the addition of $\mathrm{Mn}$ should cause an increase in the atomic volume which leads to a gradual increase of $\mathrm{T}_{N}(\epsilon)$ up to about $210 \mathrm{~K}$ for 10 at. $\% \mathrm{Mn}$, as shown by the dashed line. At larger $\mathrm{Mn}$ concentrations $T_{\backslash}(\epsilon)$ remains relatively constant at a value around $230 \mathrm{~K}$.

The relevance of FM correlations in the $\gamma$-phase of Fe on triggering the $\gamma-\alpha$ transformation was briefly referred to in section 1. This imposes the necessity to determine the nature of magnetic coupling of the correlations in the $\gamma$-phase of Fe-Mn alloys in order to understand their martensitic transformation properties. At present there are no magnetic neutron diffuse scattering experiments in the PM-state of Fe-Mn alloys in the concentration range of interest. Despite the lack of direct evidence, it is safe to assume that for temperatures just above $T_{v}(\gamma)$ the correlations are $\mathrm{AF}$, as shown in Fig. 5 . On the other hand the Fe-rich alloys with $\mathrm{x}<10$ at. $\%$ are anti-Invar which requires that the correlations in the $\gamma$-phase be FM [13]. In the region $10<x<15$ at. \% a transition region from FM to AF correlated PM-state occurs. With the loss of FM correlations the $\alpha$-phase can no longer be triggered and the $\epsilon$-phase begins to gain stability. This is very 


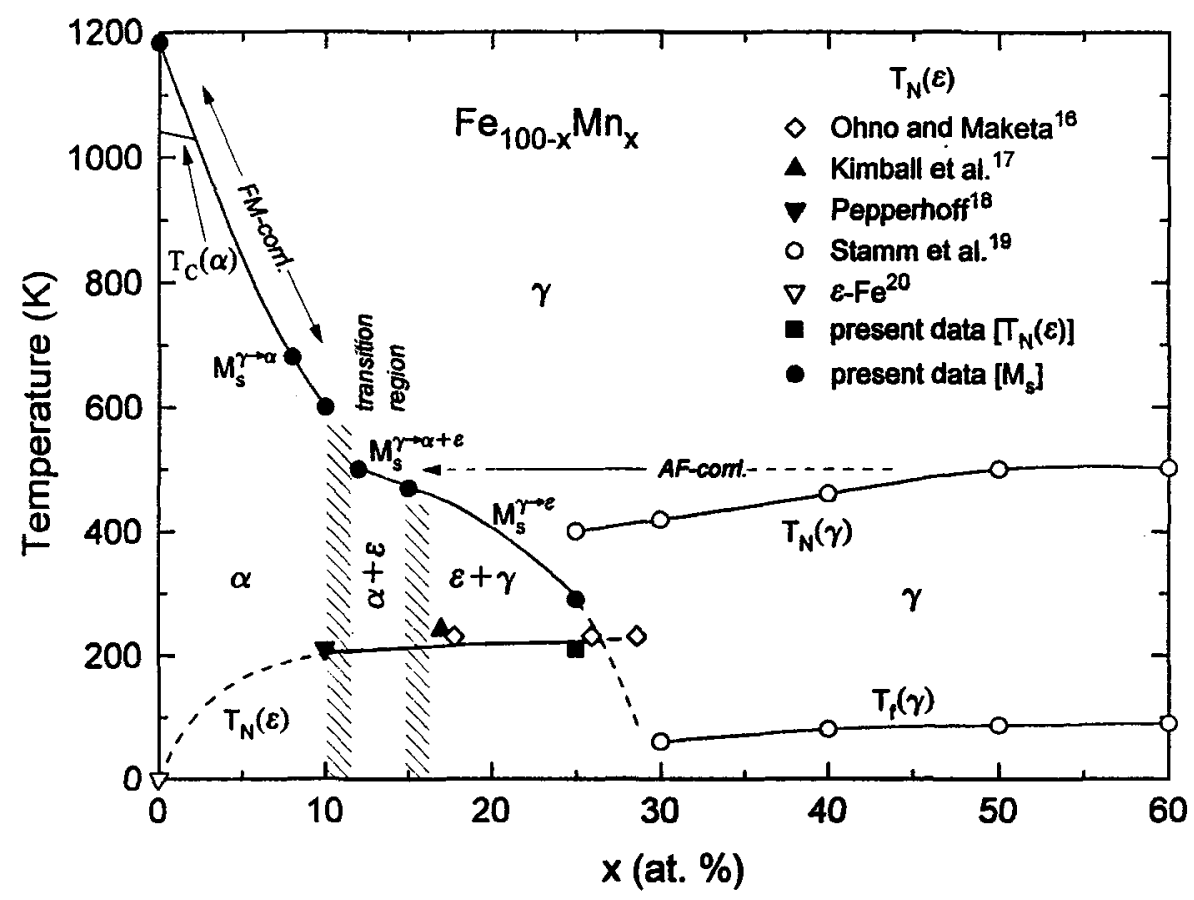

Fig. 5. The austenite to martensite transformation temperatures and the magnetic transformation temperatures of $\mathrm{Fe}_{100-x} \mathrm{Ni}_{\mathrm{x}}$. The nature of magnetic correlations (corrl)

similar to the situation in pure $\mathrm{Fe}$, where energy considerations show that the $\epsilon$-phase is more stable relative to a nonmagnetic $\alpha$-phase at all temperatures, and more stable relative to the $\gamma$-phase below about $500 \mathrm{~K}$. We note here that the $\gamma-\epsilon$ transformation temperatures within the transition region is also around $500 \mathrm{~K}$ [13]. This is not only the case for the presently investigated $\mathrm{Fe}-\mathrm{Mn}$ alloys, but also for other binary alloys of Fe such as Fe-Ru or Fe-Ir, which also undergo a $\gamma-\epsilon$ transformation [9].

In binary alloys of $\mathrm{Fe}$ with $\mathrm{Ni}$, Pd or Pt an $\epsilon$-phase does not stabilize since FM correlations in the $\gamma$-phase remain strong enough to drive the structure into an $\alpha$-phase up to around 70 at. $\% \mathrm{Fe}$. A further interesting example which serves as a support for the present arguments is the $\mathrm{Fe}-\mathrm{Co}$ alloy system. Unlike $\mathrm{Ni}, \mathrm{Pd}$ or $\mathrm{Pt}$, which are fcc, $\mathrm{Co}$ is hexagonal in the ground state, and therefore one would expect that the $\epsilon$-phase of $\mathrm{Fe}$ would readily stabilize in the Fe-Co alloys. However, due to strong FM correlations in the $\gamma$-phase, the alloys remain in the $\alpha$-phase in the ground state up to nearly 95 at. $\% \mathrm{Fe}$.

As a further result following the above arguments an $\epsilon \rightarrow \alpha$ or an $\epsilon+\alpha \rightarrow \alpha$ transformation with a change in temperature at a fixed concentration is not possible in Fe-Mn alloys. For this reason the boundaries separating the $\alpha+\epsilon$-phase from the $\alpha$ and $\alpha+\gamma$-phases are drawn vertically. In earlier literature where the influence of magnetic properties of the alloys on the transformations were not considered an $\alpha-\epsilon$ transformation with changing temperature was considered to be possible [9].

As a concluding remark we note that the stability of the $\alpha, \gamma$, or $\epsilon$-phases of Fe-rich binary Fe-alloys is esentially a problem of the stability of $\mathrm{Fe}$ in any one of these environments.

\section{Acknowledgements}

We would like to thank W. Pepperhoff for the availability of his unpublished Mössbauer experiment results on $\epsilon-\mathrm{Fe}-\mathrm{Mn}$ and and $\mathrm{G}$. Frommeyer for some of the samples used in the present experiments, and for their continued interest. This work was supported by the Deutsche Forschungsgemeinschaft (SFB 166). 


\section{References}

[1] Uhl M., Sandratskii L. M. and Kübler J., J. Magn. Magn. Mater. 103 (1992) 314-324.

[2] Moruzzi V. L., Marcus P. M. and Kübler J., Phys. Rev. B 39 (1989) 6957-6961.

[3] H. Duschanek, Mohn P. and Schwarz K., Physica B 161 (1989) 139-142.

[4] Wassermann, E. F., in Ferromagnetic Materials V (North Holland, Amsterdam, 1990) pp. 237-322.

[5] Acet M., Schneider T., Zähres H., Wassermann E. F. and Pepperhoff W., Phys. Rev B 49 (1994) $7015-$ 7020 .

[6] Hansen M., Constitution of Binary Alloys (McGraw-Hill, New York, 1958) pp. 664-668

[7] Ishikawa

[8] Schneider T., Acet M., Rellinghaus B., Wassermann E. F. and Pepperhoff W., Phys. Rev. B 151 (1995) 8917-8921.

[9] Schumann H., Neue Huitte 5 (1966) 299-303, Z. Metallkde. 58 (1967) 207-210, Arch. Eisenhüttenves. 8 (1967) 647-656.

[10] Cotes S., Baruj A., Sade M. and Guillermet A. F., J. Phys. IV Colloq. C2 5 (1995) 83-88.

[11] Tamarat K., Escorne M., Percheron-Guegan A. and Dubois B., J. Phys. IV Colloq. C2 5 (1995) 89-94.

[12] Sato A., Soma K., Chishima E. and Mori T., Acta Metall. 30 (1982) 1177-1183.

[13] Acet M., Schneider T. and Wassermann E. F., J. Phys. IV Colloq. C2 5 (1995) 105-109.

[14] Bendick W. and Pepperhoff W., Acta Metall. 30 (1982) 679-.

[15] Federzoni L. and Guénin G., J. Phys. IV Colloq. C2 5 (1995) 525-530.

[16] Ohno H. and Mekata M., J. Phys. Soc. Japan 31 (1971) 102-108.

[17] Kimball C., Gerber W. D. and Arrott A., J. Appl. Phys 34 (1963) 1046-1047.

[18] Pepperhoff, W., unpublished.

[19] Stamm W., Zähres H., Acet M., Schletz K. and E. F. Wassermann, J. Phys Colloq. C8 49 (1988) 315316.

[20] $\epsilon-F e T_{x}$

[21] Podgorny, M., $\epsilon-\mathrm{Fe}$

[16] Brown P. J., Jassim I. K., Neumann K. -U. and Ziebeck K. R. A., Physica B 161 (1989) 9-16.

[15] Bundy, F. P., J. Appl. Phys. 36 (1965) 616-620.

Acet M., Zähres H., Stamm W., Wassermann E. F. and Pepperhoff W., Physica B 151 (1989) 67-71.

Garstein E. and Rabinkin A., Acta Metallurgica 27 (1979) 1053-1064.

Lowde R. D., Harley R. T., Saunders G. A., Sato M., Scherm R. and Underhill C., Proc. R. Soc. Lond. A 374 (1981) 87-114.

Frommeyer 